\title{
CAN CONTINUOUS, INTER-GENERATIONAL COOPERATION POSITIVELY IMPACT? FINAL RESULT
}

\author{
R. Rokkaku', A. Homma², S. Kobayashi ${ }^{1}$ Y. Seki ${ }^{1}$
}

\begin{abstract}
An aim of the present study is to examine the impact of inter-generational cooperation on the quality of life of elderly Alzheimer's sufferers. The study is a continuing, two-year intervention report. The subject consist of an intervention and a control groups of six and five sufferers, respectively, who were diagnosed with Alzheimer's disease. Both groups attend day care services. The intervention group participates in the inter-generational program with children, while the control group does not. In the results, the score of Quality of Life - Alzheimer's disease (QOL-AD) of the subjects has been significantly higher in the intervention group comparing with that of the control group. been significantly higher in the intervention group comparing with that of the control group. Also the Philadelphia Geriatric Center Affect Rating Scale(PGC-ARS), have been significantly higher in the intervention group those in the control group., The magnitude of the change was not so remarkable as to influence QOL-AD at home. The present intergenerational cooperation may improve the quality of life of moderate to severe Alzheimer's sufferers.
\end{abstract}

Key words: Inter-generational cooperation, Alzheimer's sufferers, children, quality of life.

\section{Introduction}

In Japan over recent years, a rising number of nuclear family households means fewer children live with elderly relatives. Between 1975 and 2010, the ratio of threegeneration households, those with a head-of-household 65 years or older, relative to all other types of households, has steadily fallen from 11.8 to $6.9 \%$ (1). Therefore, interaction between children and senior citizens has been decreasing. Moreover, it seems that socio-economic conditions have changed in Japan, requiring young adults to relocate for work. This creates various problems that the elderly generation traditionally provides, such as emotional support, mentoring, babysitting services, and the teaching of customs to the youth generation.

Although a few results have been reported on the intergenerational program between the elderly persons and children, no results have been reported on the inter-generational cooperation between elderly persons with Alzheimer's disease and children. An aim of the present study is to clarify the effect of an intergenerational program for Alzheimer's sufferers through

1. Tokyo University of Technology, Tokyo, Japan; 2. Dementia care research and training center of Tokyo, Japan

Corresponding Author: Ryoko Rokkaku, Ph.D., R.N. School of of Nursing, Dokkyo Medical University, Kitakobayashi Mibu-Machi, Shimotsuga-Gun Tochigi, Japan, rrokkaku@dokkyomed.ac.jp, Tel +81 282-86-1111(5188) the cooperation with children. This is a final report of the results, two-year intervention from June 2012 to June 2014.

\section{Subjects}

The subjects of the study consist of the intervention group of eight Alzheimer's sufferers and six Alzheimer's sufferers for the control group, all of whom have been diagnosed with dementia of the Alzheimer's type, based on the diagnostic criteria of DSM-IV-TR (2). Informed consent to participate in the study was obtained from the participants themselves, their carers, and the parents of the children. The intervention group attends day care services at one site in city $\mathrm{A}$ and participates in an inter-generational program with children. The control group attends services at another site, also in city A, and is not given the opportunity to interact with children. Both sites are operated by the same organization and the care staff of the both sites rotates at regular intervals to minimize the difference of care. Also, the condition of formal care services is strictly defined by the Long-Term Care Insurance Law in Japan. 
Table 1

Mean age and the scores of FAST, HDS-R, Behave-AD, DAD and QOL-AD at the baseline and 12 months and 24 months in the intervention and the control groups

\begin{tabular}{llcccccccc}
\hline & & No & Mean Age & FAST & HDS-R & Hehave-AD & DAD & QOL-AD (P) & QOL-AD (F) \\
\hline Intervention group & Baseline & 11 & 86.5 & 4 & 12.18 & 1.18 & 52.7 & 35.27 & 31.36 \\
& 12 months & 8 & 88.1 & 5.62 & 12.75 & 1.38 & 43.2 & 36.25 & 32 \\
& 24 months & 6 & 88.1 & 5.17 & 11.67 & 1.17 & 42 & 35.4 & 27.5 \\
\multirow{3}{*}{ Control group } & Baseline & 12 & 84.5 & 4.56 & 9.37 & 1 & 35.6 & 32.08 & 29.92 \\
& 12 months & 6 & 85 & 5.83 & 10.33 & 1.17 & 28.8 & 30.17 & 28 \\
& 24 months & 5 & 84 & 5.6 & 7.6 & 0.8 & 15.1 & 31 & 26.4 \\
\hline
\end{tabular}

Table 2

Philadelphia Geriatric Center-Affect Rating Scale: The scores of the baseline and 12 months and 24 months in the intervened group and the control group

\begin{tabular}{|c|c|c|c|c|c|c|c|c|}
\hline & & No & Pleasure & Anger & $\begin{array}{l}\text { Anxiety/ } \\
\text { Fear }\end{array}$ & $\begin{array}{c}\text { Depression/ } \\
\text { Sadness }\end{array}$ & Interest & Contentment \\
\hline \multirow[t]{2}{*}{ Intervention group } & Baseline & 11 & 4.55 & 1.18 & 1.45 & 1.09 & 4.73 & 4.45 \\
\hline & 24 months & 6 & 5 & 1.33 & 1.5 & 1 & 5 & 5 \\
\hline Control group & Baseline & 12 & 4.83 & 1 & 1 & 1 & 4.83 & 5 \\
\hline
\end{tabular}

\section{Methods}

The program consists of activities held every morning from 10 to 11:30, with such traditional crafts as making paper fans, carp streamers, rice cakes, toys, and dolls, singing traditional songs, and playing traditional games. All activities are planned one month in advance with the requirements that they are fun, easy-to-do, continuous and varied. The care staff are all nationally licensed nurses, care workers, care helpers, or nursery school teachers.

Assessment are made every six months using the following scales: FAST (Functional Assessment Staging) (3) to measure severity of dementia, HDS-R (Hasegawa's Dementia Scale, revised) (4) which is similar to MMSE and the most common simple psychometric scale in Japan, DAD (Disability Assessment for Dementia) (5) to measure ADL (activities of daily living), BehaveAD (Behavioral Pathology in Alzheimer's Disease) (6) to measure behavioral complications, PGC-ARS (Philadelphia Geriatric Center-Affect Rating Scale) (7) to measure the status of the mood, and QOL-AD (Quality of Life in Alzheimer's Disease) (8) to measure quality of life. A nurse administered HDS-R and evaluated FAST, DAD, Behave-AD, and QOL-AD based on the information of family carers. Also, the same nurse assessed PGC-ARS from the facial expressions and behaviors of the subjects.

In the analysis, the total scores of six scales were analyzed by analysis of variance between the groups. The main effects between the groups, interacting effects by time and the group and the items of DAD, Behave-AD, PGC-ARS and QOL-AD were also analyzed.

Regarding ethical considerations, the IRB of the Tokyo University of Technology approved the protocol of the study.

\section{Results}

Eight subjects of the intervened group and six subjects of the control group were assessed after 12 months and 24 months. The demographics of the subjects, including mean age, severity of dementia, FAST, HDS-R scores, Behave-AD, DAD, QOL-AD(Family), at the baseline and 12 months and 24 months of the intervened group and the control group are shown in Table 1. No significant differences were found at the baseline between the intervention and the control groups. In the results of analysis of variance, no significant main effects of the group were found in the scores of FAST, HDS-R, DAD, Behave-AD and QOL-AD(Family) between the two groups.

As to QOL-AD(sufferer), we find that the Interventionsufferers' levels have remained basically stable. Controlsufferers QOL-level, however, has fallen slightly. Household results did not result in any significant differences. 


\section{QOL-AD(Patient)}

As to PGC-ARS, six items of the scale were separately analyzed. Although no significant main effect of the group were not found in the six items, significant interactive effects by the group and time were obtained in the items of pleasure $(\mathrm{F}(1,13)=9.180(\mathrm{p}<0.01))$, interest $(\mathrm{F}$ $(1,13)=8.630(\mathrm{p}<0.01))($ Table 2, Fig. 1, Fig. 2) .

Figure 1

QOL-AD Sufferers' QOL

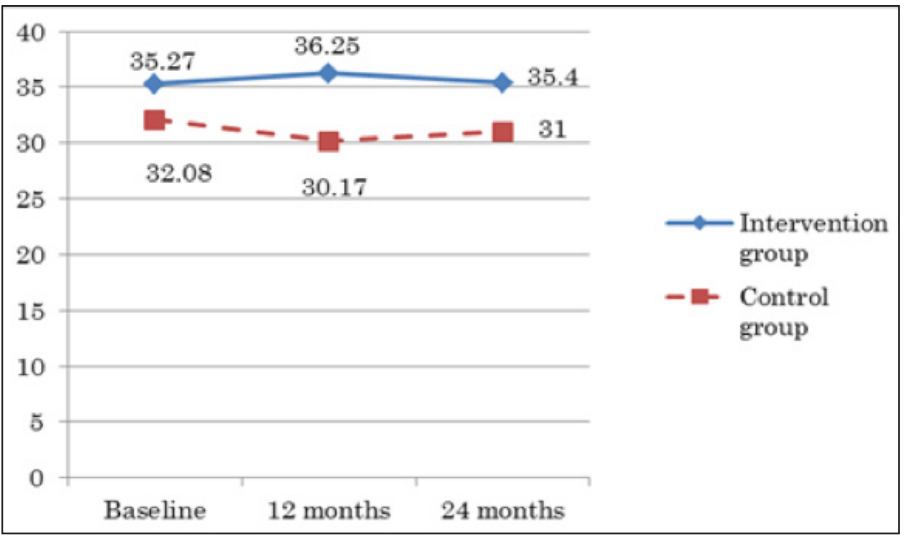

${ }^{* *}>.01$

(1) pleasure, for example smiling, laughing, friendly touching, nodding one's head, etc., remained quite stable over the past year for the Intervention Group, while those in the Control Group demonstrated a drop in such behaviors.

and (2) interest, which is identified by such signs as following a moving object with the eyes, concentrated visual focus on a person or object, stable eye contact, and physical or oral reaction to stories or music.

In the Control Group, baseline levels for Pleasure and Interest are both 4.83. However, after 12 months, the levels fell to 2.67 and 3.50 and rose again to 3.20 and 4.00 , respectively. On the other hand, in the Intervention group, Pleasure levels at the baseline, after 12 months, and after 24 months changed from 4.55 to 4.50 then rose to 5.00, respectively. In terms of Interest, the levels consistently rose from 4.73 to 4.75 to 5.00 .

In other words, the Intervention group had statistically significant increases in Pleasure and Interest over the Control group.

In addition, Moreover, the elderly have shown changes in their interactions, related to the children.

- They smile more.

- They display affection, such as hugging, holding hands, and stroking the heads of the children.

- They have started taking care of the children, for example grooming or cleaning them.

Surprisingly,

- They call the children by name.and

- In conversations among the elderly sufferers, they discuss the children much more.

\section{Figure 2}

Philadelphia Geriatric Center Affect Rating Scale: Pleasure

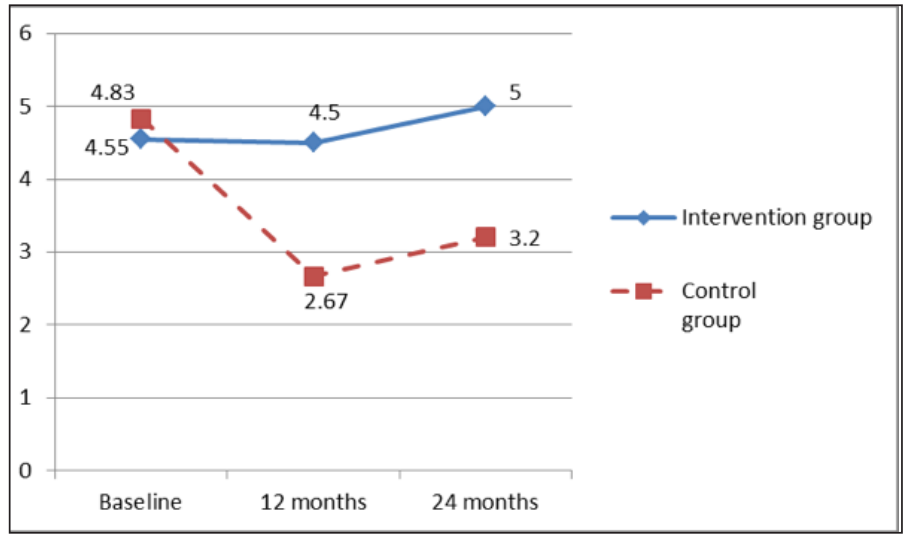

${ }^{* *}>.01$

Figure 3

Philadelphia Geriatric Center Affect Rating Scale: Interest (average score)

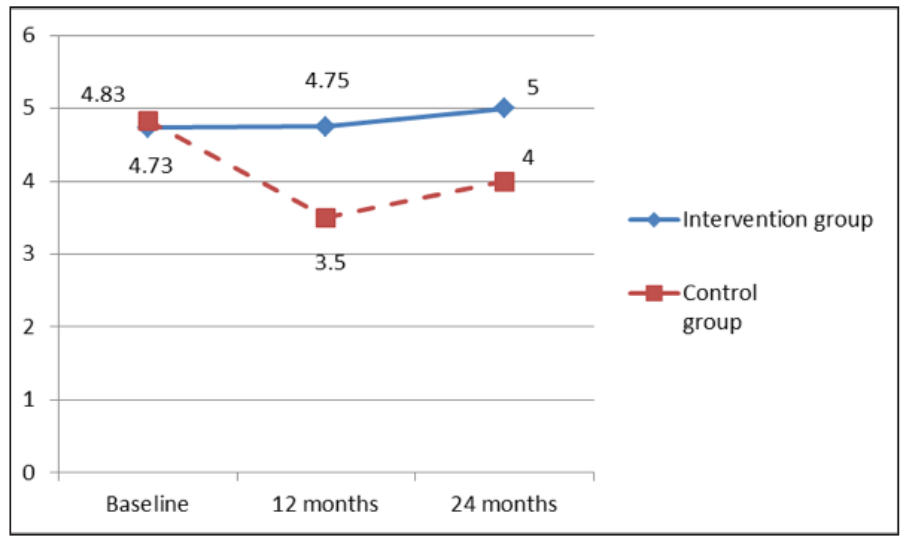

${ }^{* *}>.01$

\section{Discussion}

In the final results, and of the three evaluation scales used,

First, on the QOL-AD(Sfferes) scale, Intervention sufferers reported stable conditions, while the Control sufferers reported decreases. Second, on the Philadelphia Geriatric Center Affect Rating Scale, two items were statistically significant: Pleasure and Interest increased with inter-generational cooperation. Contact with children seems to positively affect the AD sufferers through mental stimulation.

In the results, no differences were obtained in HDS$\mathrm{R}, \mathrm{DAD}$, Behave-AD and QOL-AD(Family) between the groups. DAD, Behave-AD and QOL-AD(Family) were evaluated based on the information from carers. So this means the program had no psychosocial effects 
at home for the both groups regardless of number of uses of day services. However, significant interactive effects were obtained in the items of Pleasure, Interest of PGC-ARS. This implies that the positive affect of the intervened group has been maintained rather than the control group while attending the sessions in the day care service. This may reduce the burden on the staff caring for Alzheimer's sufferers in the day care services.

The present results have three major limitations. The first one is the small number of subjects. five and eight subjects dropped out in the intervention and the control groups, respectively, during the twenty-four months. This complicates conclusions. If the subjects are limited to those with very early stage of Alzheimer's disease, the number of subjects who dropped out would fall. However, it is difficult to access the subjects with very early Alzheimer's disease under Japan's long-term care insurance. The second limitation is that the method of the study is not a randomized controlled study. However, it is not possible to assign the subjects randomly since the inter-generational cooperation in the present study is a group program, not individual cooperation. The third limitation is that the effects to the staff, the children, and their parents are not quantitatively measured, while enlightening and educational effects to the parents of the children are apparent.

\section{Conclusion}

First, on the QOL-AD(Sfferes) scale, Intervention sufferers reported stable conditions, while the Control sufferers reported decreases .Second, on the Philadelphia Geriatric Center Affect Rating Scale, two items were statistically significant: Pleasure and Interest increased with inter-generational cooperation. Contact with children seems to positively affect the AD sufferers through mental stimulation. Also, it might be useful to educate the parents of the children, whose understanding of dementia was poor.

Acknowledgements: This study has been fully funded by the Japanese Ministry of Education, Culture, Sports, Science and Technology. The authors thank the Alzheimer's sufferers, children and their parents participating in this study.

\section{References}

1. National Institute of Population and Social Security Research. Vital Statistics of Japan, 2013. Available at: http:/ / www.ipss.go.jp/syoushika/tohkei/ Popular/Popular2013.asp?chap=0. Cited 6 January 2014.

2. American Psychiatric Association. Diagnostic and Statistical Manual of Mental Disorders, 4th ed. text revised. Washington D.C.: APA; 2000.

3. Sclan SG, Reisberg B. Functional Assessment Staging (FAST) in Alzheimer's disease: reliability, validity, and ordinality. International Psychogeriatrics.1992; 4 (03):55-69.

4. Katou S. Revising the Hasegawa simple intelligence evaluation scale (HDSR). Japanese Journal of Geriatric Psychiatry. 1991; 2:1339-1347.

5. Gelinas I, Gauthier L, McIntyre M. Development of a functional measure for persons with Alzheimer's disease. The disability assessment for dementia. American Journal of Occupational Therapy. 1999; 53:471-481.

6. Reisberg B, Auer SR, Monteiro IM. Behavioral pathology in Alzheimer's disease (BEHAVE-AD) rating scale. International Psychogeriatrics. 1997; 8:301-308.

7. Lawton PM. Quality of life in Alzheimer's disease. Alzheimer Disease Association Disorders. 1994; 8(3):138-150.

8. Revell AJ. Quality of life in the Alzheimer's disease (QoL-AD) scale: factor solutions in non-demented elders. University Park: Pennsylvania State University; 2002. p.5. Available at: http:/ / www.uwpsychiatry.org/sls/ QOL_AD_2002.pdf. Cited January 7, 2014.

9. Phung KTT, Waldorff FB, Buss DV, et al. A three-year follow-up on the efficacy of psychosocial interventions for patients with mild dementia and their caregivers: the multicentre, rater-blinded, randomized Danish Alzheimer Intervention Study (DAISY). BMJ Open 2013;3:e003584. doi:10.1136/bmjopen-2013-003584.

10. Carrion C, Aymerich M, Bailles E, Lopes-Bermejo A. Cognitive Psychosocial Intervention in Dementia: a Systematic Review. Dementia and Geriatric Cognitive Disorders. 2013; 36: 363-375. 